\begin{tabular}{|c|l|}
\hline Title & Second harmonic generation study on electrochemical deposition of pall ladium on a polycry stall line gold electrode \\
\hline Author(s) & A watani, Tadashi; Y agi, Ichizo; Noguchi, Hidenori; U osaki, Kohei \\
\hline Citation & $\begin{array}{l}\text { Journal of Electroanalytical Chemistry, 524-525(1-2), 184 193 } \\
\text { https:/doi.org/10.1016/50022-0728(02)00647-2 }\end{array}$ \\
\hline Issue Date & 2002-05-03 \\
\hline Doc URL & http://hdl.handle.net/2115/20567 \\
\hline Type & article (author version) \\
\hline File Information & jec524-525.pdf \\
\hline
\end{tabular}

Instructions for use 


\title{
Second Harmonic Generation Study on Electrochemical Deposition of Palladium on a Polycrystalline Gold Electrode
}

Tadashi Awatani, Ichizo Yagi, Hidenori Noguchi and Kohei Uosaki*

Physical Chemistry Laboratory, Division of Chemistry, Graduate School of Science, Hokkaido University, Sapporo 060-0810, Japan

\begin{abstract}
Electrochemical deposition (electrodeposition) and dissolution processes of $\mathrm{Pd}$ on a polycrystalline $\mathrm{Au}$ electrode surface in $\mathrm{HClO}_{4}$ solution containing $\mathrm{PdCl}_{4}{ }^{2-}$ were investigated by second harmonic generation (SHG) measurements. Both $1064 \mathrm{~nm}$ (near-IR) and $600 \mathrm{~nm}$ (visible) excitations were employed to monitor the SHG behavior in situ. Under the near-IR excitation, the resulting SHG signal contained contributions from both the $\mathrm{Pd}$ deposit and the adsorbed $\mathrm{PdCl}_{4}{ }^{2-}$. On the other hand, the adsorption of the $\mathrm{PdCl}_{4}{ }^{2-}$ did not affect the SHG signal under the visible excitation. The SHG signal under the visible excitation was resonantly enhanced with the first and second monolayer depositions of Pd but declined with higher Pd coverage. These results confirm the unique structure and property of an ultra-thin Pd overlayer on gold that was suggested by scanning tunneling microscopy (STM), surface x-ray scattering (SXS), and electrochemical measurements at electrodeposited Pd thin layers on single crystalline gold surfaces.
\end{abstract}


Keywords: Second Harmonic Generation; Gold; Palladium; Electrochemical Methods;

Electrodeposition

*Corresponding author: Fax: +81-11-706-3440

Email: uosaki@pcl.sci.hokudai.ac.jp 


\section{Introduction}

Physical and chemical properties of metal surfaces with a deposited foreign metal thin layer are known to be different from those of the substrate metal and of the deposited metal in the isolated bulk state. For example, the deposition of catalytic-active metals such as Pt, $\mathrm{Pd}$ and $\mathrm{Ru}$ on catalytic inactive noble metal surfaces significantly improves the catalytic activities for a wide variety of chemical reactions and has been well described in several review articles [1-5]. Among these catalytic-active metals, $\mathrm{Pd}$ is considered to be particularly important for industrial application and fundamental science because of its high affinity for bulk hydrogen absorption. Thus, the depositions of Pd on noble metals such as $\mathrm{Au}$ and Pt metal surfaces in both UHV [6- 12] and aqueous electrochemical environments have recently attracted considerable interests [13-16]. Although it has been generally regarded that physically deposited layers gave better ordered deposits than layers formed by electrochemical deposition [17], the recent advancements in atomic resolution imaging have shown that this was not always the case [18-20]. For example, electrochemical scanning tunneling microscopy (STM) [21-27] studies showed that Pd electrodeposition on Au single crystalline electrode proceeds in layer-by-layer mode and detailed structural investigation by surface x-ray scattering (SXS) $[28,29]$ proved that electrodeposited Pd layers developed epitaxially without Pd-Au alloy formation, following 
the crystalline orientation of the $\mathrm{Au}$ substrate both at $\mathrm{Au}(111)$ [28] and $\mathrm{Au}(100)$ [29] single crystalline electrodes. As STM and electrochemical quartz crystal microbalance (EQCM) [22] measurements have shown the adsorption of the $\mathrm{PdCl}_{4}{ }^{2-}$ complex on both the substrate gold and the deposited Pd with an ordered structure, we proposed that the ordered adlayer of the $\mathrm{PdCl}_{4}^{2-}$ complex suppresses the three-dimensional growth and leads to the two-dimensional layer-by-layer growth of the Pd layers [22,25,28].

Various techniques such as infrared absorption spectroscopy (IRAS) [30] and electrochemical techniques [31,32] have been employed to unravel electrocatalytic activities $[33,34]$ and surface characteristics $[35,36]$ of the $\mathrm{Pd} / \mathrm{Au}$ surfaces. The electrochemical characteristics of ultra-thin Pd epitaxial layers electrochemically deposited on $\mathrm{Au}(111)$ and $\mathrm{Au}(100)$ surfaces were previously found to be strongly dependent on the surface structure and the thickness of the $\mathrm{Pd}$ thin overlayers [31,37]. Although electrochemical characterizations have demonstrated that the $\mathrm{Pd} / \mathrm{Au}(111)$ and $\mathrm{Pd} / \mathrm{Au}(100)$ surfaces behave essentially like $\operatorname{Pd}(111)$ and $\operatorname{Pd}(100)$ surfaces, respectively, the formation and reduction of oxide on the Pd sub-monolayer surfaces took place at more and less positive potentials, respectively, than those on the surfaces of the Pd multi-layers or the respective $\mathrm{Pd}$ bulk. The $\mathrm{Pd} / \mathrm{Au}(100)$ electrode shows a much higher electrocatalytic activity for the oxidation of formaldehyde than that at the $\mathrm{Pd} / \mathrm{Au}(111)$ electrode. Furthermore, the highest activity for the electro-oxidation of formaldehyde was observed at 
the $\mathrm{Pd} / \mathrm{Au}(100)$ electrode when the thickness of the Pd thin overlayers was less than a monolayer. Kolb et al. also reported the thickness and crystal orientation dependence on the electrocatalytic activity of the formic acid oxidation at the Pd/Au electrodes [31]. These catalytically active behaviors of $\mathrm{Pd} / \mathrm{Au}$ surface electrodes were interpreted as a result of a change in the electronic structure upon deposition of Pd overlayers. However, there is no direct evidence for any modification of the electronic structure in the electrochemical $\mathrm{Pd} / \mathrm{Au}$ system.

Optical second harmonic generation (SHG) is a nonlinear optical process in which the frequency-doubled light is generated from a localized region where a breakdown in symmetry occurs at the interface between two adjacent centrosymmetric media [38-42]. Since the SHG signal obtained is sensitive to changes at the surface and interfaces, it has been applied to a number of systems including electrochemical systems to probe interfacial phenomenon such as surface charging, adsorption and phase transition processes under resonant and/or non-resonant conditions [40,43]. The resonantly enhanced SHG signal arises when the fundamental or $\mathrm{SH}$ photon energetically couples with an electronic transition localized at the interfaces and, therefore, SHG spectroscopy has been recognized to be a powerful probe for interfacial electronic structures [38-42]. SHG spectroscopy has been applied to study the surface electronic structure of semiconductors $[44,45]$, metals $[46,47]$ and molecular electronic transitions at interfacial boundaries [48-50]. The 
application of SHG spectroscopy to electrochemical interfaces is, however, still limited. We have applied this technique for the in situ determination of the surface electronic structure of various electrochemical systems $[51,52]$ but no reports on a surface electronic structural change during metal electrodeposition using this method has been published.

In this paper, the SHG measurements with both 1064 (near-IR) and $600 \mathrm{~nm}$ (visible) excitations were carried out to probe the changes in the surface electronic structure during the electrodeposition of Pd on a polycrystalline Au electrode surface. The effect of the $\mathrm{PdCl}_{4}{ }^{2-}$ adsorption on the SHG signals was also investigated. The origin for the change in the SHG signal during the electrodeposition process is discussed with reference to the previously reported photoemission studies [53] and theoretical calculations [54,55] for $\mathrm{Pd} / \mathrm{Au}$ systems.

\section{Experimental}

Milli-Q (Millipore) water was used to prepare the electrolyte solution with reagent grade perchloric acid $\left(\mathrm{HClO}_{4}\right.$, Wako) and potassium tetrachloropalladate(II) $\left(\mathrm{K}_{2} \mathrm{PdCl}_{4}\right.$, Aldrich), which were used as received. A polycrystalline Au disk with a diameter of $10 \mathrm{~mm}$ (Tanaka Precious Metal, purity $99.99 \%$ ) was used as the working electrode. The apparent electrode surface area was $0.28 \mathrm{~cm}^{2}$ as determined by an electrode holder made of Kel-F. The $\mathrm{Au}$ substrate was mechanically polished with alumina powder $(0.05 \mu \mathrm{m})$ till a mirror-finish was developed. This was followed by a rinse and sonication in Milli-Q water. 
Further treatment of annealing/quenching of the $\mathrm{Au}$ electrode was undertaken before transferring it to a spectroelectrochemical cell made of Kel-F, which was filled with $0.1 \mathrm{M}$ $\mathrm{HClO}_{4}$ solution. For the electrochemical measurements, a standard three-electrode configuration was employed with a Pt wire counter electrode and a $\mathrm{Ag}|\mathrm{AgCl}| \mathrm{NaCl}$ (sat) electrode used as the reference electrode.

The details of the SHG measurements are described elsewhere [56-58]. Briefly, an optical parametric oscillator (Lambda Physik, Scanmate OPPO) pumped with a frequency-tripled nanosecond Nd:YAG laser (Coherent, Infinity 40-100) was employed as the laser source for the visible excitation (SHG: $300 \mathrm{~nm})$. The fundamental $(\omega)$ light of the

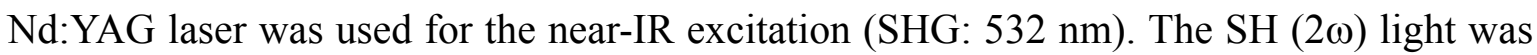
separated from the $\omega$ light using appropriate filters (Toshiba) and a monochromator (Koken Kogyo, SG-100) and was detected by a photomultiplier tube (Hamamatsu, R636-10). A digital delay generator (Stanford Research, DG535) was used to generate the trigger signals to the laser, the detection system and computer interfaces and also to control the timing of the measurements.

The SHG measurements under potential control were carried out at an incident angle of $45^{\circ}$ with an input power of $1 \mathrm{~mJ} /$ pulse and $\mathrm{p}$-in/p-out polarization at a repetition rate of $20 \mathrm{~Hz}$. The electrode potential was controlled with a potentiostat (Toho Technical Research Polarization Unit, PS-07), which was also used to provide potential modulation. The 
captured electrode potential, current and the normalized SHG intensity data were converted to digital signals by a computer interface module (Stanford Research, SR245) and sent to a personal computer (NEC, PC9801RA) via a GP-IB board (NEC, PC9801-29).

Prior to each measurement, the electrolyte solutions were deaerated by passing high-purity Ar (Air Water, purity 99.999\%) for at least $20 \mathrm{~min}$, followed by oxidation-reduction cycles between 0 to $1400 \mathrm{mV}$ in the $0.1 \mathrm{M} \mathrm{HClO}_{4}$ solution to clean the Au electrode surface. The SHG response from the clean $\mathrm{Au}$ electrode in the $0.1 \mathrm{M} \mathrm{HClO}_{4}$ solution was measured initially and then, a deaerated $0.1 \mathrm{M} \mathrm{HClO}_{4}$ solution containing the $\mathrm{PdCl}_{4}{ }^{2-}$ was introduced into the spectroelectrochemical cell with Ar pressure through a series of flow tubes without affecting the optical alignment. To prevent deposition of the $\mathrm{Pd}$ on the Au surface during the solution exchange, the electrode potential was kept at 750 $\mathrm{mV}$. After the solution exchange, the solution was purged by passing Ar for another $15 \mathrm{~min}$ and the SHG measurement was then carried out.

\section{Results}

\subsection{Cyclic Voltammetry}

The solid line in Fig. 1(a) is the cyclic voltammogram (CV) of a polycrystalline $\mathrm{Au}$ electrode in a $0.1 \mathrm{M} \mathrm{HClO}_{4}$ solution containing $0.1 \mathrm{mM} \mathrm{PdCl}_{4}{ }^{2-}$ obtained at a potential scan rate of $10 \mathrm{mV} / \mathrm{s}$. For a comparison, a typical $\mathrm{CV}$ of the polycrystalline Au electrode in a 0.1 
$\mathrm{M} \mathrm{HClO}_{4}$ solution without $\mathrm{PdCl}_{4}{ }^{2-}$ is also presented in Fig. 1(a) by the dotted line. In the solution with $\mathrm{PdCl}_{4}^{2-}$ (solid line), the cathodic current corresponding to the reductive deposition of the Pd started to flow as soon as a negative potential sweep was started from $750 \mathrm{mV}$. A cathodic peak was observed at $610 \mathrm{mV}$ and the limiting current showing the diffusion-limited electrodeposition of Pd occurred as the potential became more negative. In the positive potential scan from $100 \mathrm{mV}$, the cathodic current corresponding to the diffusion-limited electrodeposition of Pd still continued up to $500 \mathrm{mV}$. From this potential, the anodic current due to Pd dissolution flowed and an anodic current peak was observed at $710 \mathrm{mV}$. These behaviors are essentially the same as those previously reported before at gold single crystal electrodes [22]. It has been shown that the electrodeposition of Pd onto the Au electrode surface occurs via the following two-electron reduction process [22]:

$$
\mathrm{PdCl}_{4}^{2-}+2 \mathrm{e}^{-} \rightarrow \mathrm{Pd}+4 \mathrm{Cl}^{-}
$$

From the charge that flowed during the anodic dissolution of $\mathrm{Pd}$, the amount of deposited $\mathrm{Pd}$ in this particular potential cycle was calculated to be equivalent to approximately 2 monolayers (ML).

\subsection{SHG response under $1064 \mathrm{~nm}$ (near-IR) excitation}

The solid line in Fig. 1(b) shows the SHG response simultaneously obtained during a potential scan in the $0.1 \mathrm{M} \mathrm{HClO}_{4}$ aqueous solution containing $0.1 \mathrm{mM} \mathrm{PdCl}_{4}{ }^{2-}$ at an 
excitation wavelength of $1064 \mathrm{~nm}$ (SHG: $532 \mathrm{~nm}$ ). For a comparison, the potential dependent SHG response during a potential scan in the $0.1 \mathrm{M} \mathrm{HClO}_{4}$ solution without $\mathrm{PdCl}_{4}{ }^{2-}$ is shown in the same figure by the dotted line. The latter result is in good agreement with the previously published results $[59,60]$. The SHG intensity at $750 \mathrm{mV}$ was higher in the solution with $\mathrm{PdCl}_{4}{ }^{2-}$ (solid line) than that in the solution without $\mathrm{PdCl}_{4}{ }^{2-}$ (dotted line) as shown in Fig. 1(b). As the potential was swept from $750 \mathrm{mV}$ in the negative direction, the SHG signal decreased until the potential became $350 \mathrm{mV}$. It should be noted here that almost one ML of Pd deposition was completed when this potential was reached. The SHG signal was almost constant as the potential was scanned more negatively to $100 \mathrm{mV}$ and in the positive potential sweep to $550 \mathrm{mV}$. From $550 \mathrm{mV}$, an anodic current corresponding to $\mathrm{Pd}$ dissolution started to flow and the SHG signal increased accordingly. The SHG signal further increased during the negative potential sweep to the initial potential $(750 \mathrm{mV})$ for the completion of the potential cycle but did not restore the value initially observed at this potential.

Figure 2 shows the time dependences of (a) the potential, (b) the current and (c) the SHG signal at the Au electrode in the $0.1 \mathrm{M} \mathrm{HClO}_{4}$ solution containing $0.1 \mathrm{mM} \mathrm{PdCl}_{4}{ }^{2-}$ over two potential cycles. The SHG intensity at the initial potential, i.e., $750 \mathrm{mV}$, after the first potential cycle was smaller than the initial value. The second potential cycle was initiated soon after the completion of the first potential cycle. Although the time 
dependencies of the SHG signal and current in the second potential cycle were similar to those obtained in the first cycle, it is clear that the SHG intensity at a given potential was smaller in the second cycle. The SHG intensity at the initial potential, i.e., $750 \mathrm{mV}$, after the second potential cycle was smaller than that before the second potential cycle but it increased and returned to the value obtained before the second potential cycle if the potential was held at this potential for a couple of minutes. A larger time duration was, however, required for the complete restoration of the SHG intensity to the original value obtained before the first potential cycle.

\subsection{SHG response under $600 \mathrm{~nm}$ (visible) excitation}

The solid line in Fig. 1(c) shows the SHG response obtained during a potential scan in the $0.1 \mathrm{M} \mathrm{HClO}_{4}$ solution containing $0.1 \mathrm{mM} \mathrm{PdCl}_{4}{ }^{2-}$ at an excitation wavelength of 600 nm (SHG: $300 \mathrm{~nm}$ ). For a comparative purpose, the potential dependent SHG response during the potential scan in the $0.1 \mathrm{M} \mathrm{HClO}_{4}$ solution without $\mathrm{PdCl}_{4}{ }^{2-}$ is also shown in Fig. 1(c) by the dotted line. This result was totally different from the one obtained under near-IR excitation.

While the SHG intensity at $750 \mathrm{mV}$ was higher in the solution with $\mathrm{PdCl}_{4}{ }^{2-}$ (solid line) than that in the solution without $\mathrm{PdCl}_{4}{ }^{2-}$ (dotted line) under near-IR excitation as shown above (Fig. 1(b)), the presence of $\mathrm{PdCl}_{4}{ }^{2-}$ in the solution had almost no effect on the SHG 
intensity at $750 \mathrm{mV}$ under visible excitation. A time dependent SHG measurement carried out at $750 \mathrm{mV}$ by injecting concentrated $\mathrm{PdCl}_{4}{ }^{2-}$ solution into the $0.1 \mathrm{M} \mathrm{HClO}_{4}$ solution showed no change in the SHG intensity upon the addition of $\mathrm{PdCl}_{4}{ }^{2-}$, confirming no noticeable effect of the presence of $\mathrm{PdCl}_{4}{ }^{2-}$ on the $\mathrm{SHG}$ behavior under visible excitation.

As soon as the negative sweep was started from $750 \mathrm{mV}$, the SHG intensity sharply increased until ca. $600 \mathrm{mV}$ where the cathodic current peak was observed, in contrast to the fact that the SHG intensity under near-IR excitation decreased as the negative sweep was started. The SHG intensity under visible excitation further increased but only slightly until the potential scan was reversed at $100 \mathrm{mV}$. The SHG intensity at this potential in the solution with $\mathrm{PdCl}_{4}{ }^{2-}$ was much higher than in the solution without $\mathrm{PdCl}_{4}{ }^{2-}$ under visible excitation. The opposite was true under near-IR excitation. In the positive potential sweep, the SHG signal showed a steady decline up to ca. $680 \mathrm{mV}$ and then very sharply decreased, while cathodic current due to $\mathrm{Pd}$ deposition flowed between $100 \mathrm{mV}$ and $500 \mathrm{mV}$ and anodic current due to $\mathrm{Pd}$ dissolution flowed between $500 \mathrm{mV}$ and $680 \mathrm{mV}$. It is interesting to note that the sharp decline in the SHG intensity did not start as soon as the anodic dissolution started but at a much more positive potential. As soon as the potential was returned to $750 \mathrm{mV}$, i.e., upon the completion of the potential cycle, the SHG intensity returned to the initial value observed before the potential cycle was started.

The above results showed that the relation between the SHG intensity and the amount 
of deposited Pd was not simple. Figure 3 shows the time dependence of (b) the current and (c) the SHG intensity at the $\mathrm{Au}$ surface in a $0.1 \mathrm{M} \mathrm{HClO}_{4}$ solution containing $0.5 \mathrm{mM}$ $\mathrm{PdCl}_{4}{ }^{2-}$ under visible excitation when the potential was swept from 750 to $450 \mathrm{mV}$ at a sweep rate of $5 \mathrm{mV} / \mathrm{s}$ and then held at the latter potential for $30 \mathrm{~min}$ to facilitate bulk $\mathrm{Pd}$ deposition as shown in Fig. 3(a). The concentration of $\mathrm{PdCl}_{4}{ }^{2-}$ was increased to $0.5 \mathrm{mM}$ to ensure bulk Pd deposition in the timescale of the measurements. As soon as the potential was negatively swept from $750 \mathrm{mV}$, a cathodic current flowed and the SHG intensity increased. The cathodic current reached a peak (ca. $32 \mu \mathrm{A}$ ) $45 \mathrm{~s}$ after the potential scan was initiated while the maximum SHG intensity was observed after ca. 2 min. An almost constant current flowed when potential was held at $450 \mathrm{mV}$, suggesting that the diffusion-limited deposition proceeded. The SHG intensity declined with time and it became lower than that at time zero, i.e., SHG from bare gold, after ca. $20 \mathrm{~min}$.

\section{Discussion}

\subsection{General treatment for the contribution of adsorbate on the SHG signal}

The SHG response from a metal surface has been treated in detail by several authors

$[38,39,61,62]$. Under the electric dipole approximation, the SHG intensity, $I^{2 \omega}$, from a metal surface can be related to the second order surface susceptibility tensor $\left(\chi_{\mathrm{S}}^{(2)}\right)$ and the incident excitation intensity, $I^{\omega}$, as: 


$$
I^{2 \omega} \propto\left|\mathbf{e}(2 \omega) \cdot \chi_{\mathrm{S}}^{(2)} \cdot \mathbf{e}(\omega) \cdot \mathbf{e}(\omega)\right|\left(I^{\omega}\right)^{2}
$$

where $\mathbf{e}(\omega)$ and $\mathbf{e}(2 \omega)$ are the input and output light fields polarization vectors, respectively. In principle, the surface susceptibility tensor, $\chi_{\mathrm{S}}^{(2)}$, consists of 18 independent elements, $\chi_{\mathrm{ijk}}$, where $\mathrm{i}$ is the polarization vector for the $\mathrm{SH}$ beam and $\mathrm{j}$ and $\mathrm{k}$ are the polarization vector for the fundamental beam $[38,41]$. By taking surface symmetry into account, the number of the non-zero tensor elements can be reduced. For an isotropic surface, i.e. polycrystalline $\mathrm{Au}$ surface, the non-zero susceptibility tensor elements are $\chi_{z z z}, \chi_{z x x}$ and $\chi_{x x z}$. The $\chi_{z x x}$ and $\chi_{x x z}$ elements can be monitored by the SHG measurements in the s-in/p-out and 45-in/s-out polarization configurations, respectively. For p-in/p-out polarization, on the other hand, all three nonzero tensor elements are reflected in the SHG signal. From the polarizations dependence studies on the $\mathrm{Pd} / \mathrm{Au}$ surface, the large change in the SHG signal, during Pd deposition on Au surface, was only observed in the $\mathrm{p}$-in/p-out polarization condition, hence, the dominant tensor element was specified to the $\chi_{z z z}$ element [63]. Therefore, in this paper, the SHG response is assumed to mainly arise from change in the $\chi_{z z z}$ element.

With the presence of an adsorbate, additional susceptibility terms are required to describe its influence. The new modified surface susceptibility tensor with an adsorbate, $\chi_{\mathrm{S}}^{(2)^{\prime}}$, is given by:

$$
\chi_{\mathrm{S}}^{(2)^{\prime}}=\chi_{\mathrm{S}}^{(2)}+\chi_{\mathrm{A}}^{(2)}+\Delta \chi_{\mathrm{I}}^{(2)}
$$


where, $\chi_{\mathrm{S}}^{(2)}, \chi_{\mathrm{A}}^{(2)}$ and $\Delta \chi_{\mathrm{I}}^{(2)}$ are the nonlinear susceptibility tensor of the substrate, the inherent nonlinear susceptibility tensor of the adsorbate, and the perturbation in the nonlinear susceptibility tensor due to the interaction between the adsorbate and the substrate surface, respectively.

\subsection{Effect of $\mathrm{PdCl}_{4}{ }^{2-}$ adsorption on the SHG signal}

The SHG signals obtained under both excitation wavelengths from the Au electrode surface in the $\mathrm{HClO}_{4}$ solution are known to be resonantly enhanced $[59,60]$. A resonant SHG under near-IR excitation occurs as a result of the resonance of the $2 \omega\left(2 \omega_{I R}=2.33\right.$ $\mathrm{eV}$ ) photon with either the $5 \mathrm{~d}-6 \mathrm{~s}$ interband transition of $\mathrm{Au}$, positioned at $2.25 \mathrm{eV}$ [64] or from intraband transition within the sp band [65]. On the other hand, the $2 \omega$ photon generated under visible excitation $\left(2 \omega_{\mathrm{VIS}}=4.13 \mathrm{eV}\right)$ can be resonant with multiple transitions from the deeper states of the d-band to the upper states above the Fermi level, so that the SHG signal behavior should be complex and sensitive to the bound electronic structure [59].

At $750 \mathrm{mV}$, where neither a cathodic nor anodic current flowed, the SHG intensity under near-IR excitation was larger in the solution with $\mathrm{PdCl}_{4}{ }^{2-}$ than in the solution without $\mathrm{PdCl}_{4}{ }^{2-}$, as shown in Fig. 1(b) but almost no effect of the $\mathrm{PdCl}_{4}{ }^{2-}$ on the SHG signal was observed under visible excitation (Fig. 1(c)). We have already reported in that $\mathrm{PdCl}_{4}{ }^{2-}$ was 
adsorbed on the Au single crystalline electrodes at this potential and its adsorption plays a crucial role in the layer-by-layer growth of the $\mathrm{Pd}$ overlayer [22,25]. Under this circumstance, i.e., with the $\mathrm{PdCl}_{4}{ }^{2-}$ adsorbate layer, Eq. 2 can be written as:

$$
\chi_{\mathrm{S}}^{(2)^{\prime}}=\chi_{\mathrm{Au}}^{(2)}+\chi_{\mathrm{PdCl}_{4}^{2-}}^{(2)}+\Delta \chi_{\mathrm{PdCl}_{4}^{2-}-\mathrm{Au}}^{(2)}
$$

where $\chi_{\mathrm{Au}}^{(2)}$ is the susceptibility of the Au surface without any adsorbate, $\chi_{\mathrm{PdCl}_{4}^{2-}}^{(2)}$ is the susceptibility of $\mathrm{PdCl}_{4}^{2-}$ and $\Delta \chi_{\mathrm{PdCl}_{4}^{2-} \text { Au }}^{(2)}$ represents the perturbation in the nonlinear susceptibility due to the interaction between the adsorbed $\mathrm{PdCl}_{4}{ }^{2-}$ and the $\mathrm{Au}$ substrate.

Although a detailed discussion on the origin of the effect of the $\mathrm{PdCl}_{4}{ }^{2-}$ adsorption on the SHG activity is not possible from the available data at this stage, the excitation wavelength dependent SHG intensity change caused by the adsorption of $\mathrm{PdCl}_{4}{ }^{2-}$ provides some clues. The fact that the existence of $\mathrm{PdCl}_{4}{ }^{2-}$ has a significant impact on the $\mathrm{SHG}$ signal under near-IR excitation but has a negligible effect under visible excitation suggest that the last two terms of Eq. 3, i.e., $\chi_{\mathrm{PdCl}_{4}^{2-}}^{(2)}$ and/or $\Delta \chi_{\mathrm{PdCl}_{4}^{2-}-\mathrm{Au}}^{(2)}$, are important under near-IR excitation but negligible under visible excitation. The contribution of the former term should be small as the adsorbed $\mathrm{PdCl}_{4}{ }^{2-}$ forms a two-dimensionally ordered adlayer that is $\mathrm{SHG}$ inactive. The change of $\Delta \chi_{\mathrm{PdCl}_{4}^{2-}-\mathrm{Au}}^{(2)}$ associated with $\mathrm{PdCl}_{4}{ }^{2-}$ adsorption may be caused by the modifications of bound electronic structure, surface charge density and the nonlinear polarizability of surface free electrons.

The near-IR excited SHG signal $\left(2 \omega_{\mathrm{IR}}=2.33 \mathrm{eV}\right)$ should probe electronic structure in 
the energy region shallower than that under visible excitation $\left(2 \omega_{\mathrm{VIS}}=4.13 \mathrm{eV}\right)$, which probes deeper in the d-band energy levels. If chemical bonding is formed between the adsorbed $\mathrm{PdCl}_{4}{ }^{2-}$ and the $\mathrm{Au}$ surface, a significant change in the electronic structure involving the d-band is expected. There are reports that showed the adsorption of alkanethiol on Au lead to a significant changes in the SHG intensities under both near-IR and visible excitations $[66,67]$, showing a d-band structural change as a result of S-Au chemical bond formation [68]. However, in the present case, the change in the SHG intensity was not significant and only observed under near IR excitation. Thus, the $\mathrm{PdCl}_{4}{ }^{2-}$ adsorption was not too strong to modify the electronic structure involving the d-band.

Modification of the surface charge density and the nonlinear polarizability of the surface free electrons on the $\mathrm{Au}$ electrode surface would be expected with adsorption of $\mathrm{PdCl}_{4}{ }^{2-}$. Both of these modifications can perturb the SHG signal. In the former case, if the potential of zero charge (PZC) of the electrode surface shifts to more negative values with adsorption of $\mathrm{PdCl}_{4}^{2-}$, electric field becomes stronger, resulting in the enhancement of the SHG signal. This enhancement effect is commonly known as electric field induced SH (EFISH) [40]. This effect was found not to be as pronounced on the $\mathrm{Au}$ as on the $\mathrm{Ag}$ electrode surfaces under both near-IR and visible excitations [59]. This is because the EFISH effect on the Au electrode surface is smaller than the $\chi_{\mathrm{Au}}^{(2)}$ contribution. Therefore, the charge density modification associated with $\mathrm{PdCl}_{4}{ }^{2-}$ adsorption on the Au electrode did 
not directly correspond to the observed increase in the SHG signal. The modification of the nonlinear polarizability of the surface free electrons also influences the SHG signal. Since the near-IR excited light can probe a nonlinear response of the surface free electrons, especially near the Fermi level, more than the visible excited light, the influence becomes more significant under near-IR excitation. Thus, considering the wavelength dependent SHG behavior with $\mathrm{PdCl}_{4}{ }^{2-}$ adsorption, the increase in the SHG signal observed with $\mathrm{PdCl}_{4}{ }^{2-}$ adsorption should be related to the modification of the nonlinear polarizability of the surface free electrons.

Based on the above considerations, the weaker SHG intensity at $750 \mathrm{mV}$ after the completion of the first potential cycle under near-IR excitation (Fig. 1(b)) while equal in the case of visible excitation (Fig. 1(c)) can be explained as follows. As the potential was swept towards positive potential, the Pd deposit dissolved and consequently the lift of the adsorbed $\mathrm{PdCl}_{4}{ }^{2-}$ from the surface took place. When the potential scan was stopped, some time duration was required for the readsorption of the $\mathrm{PdCl}_{4}{ }^{2-}$ back onto the Au surface. As a result, a weaker SHG response was obtained under near-IR excitation since the SHG signal reflects the amount of adsorbed $\mathrm{PdCl}_{4}{ }^{2-}$ on the $\mathrm{Au}$ surface as suggested above. The slow recovery of the SHG signal under near-IR excitation to the original value should be due to the slow readsorption kinetics of $\mathrm{PdCl}_{4}{ }^{2-}$. No difference in the SHG intensity was expected under visible excitation between before and after the first or second potential 
cycle because the SHG signal is not significantly affected by the adsorption of $\mathrm{PdCl}_{4}{ }^{2-}$ as mentioned above.

\subsection{Pd coverage dependence on the SHG signal}

\subsubsection{Initial stages of Pd deposition (0 to 3 ML Pd deposition)}

The coverage dependence of SHG can give information regarding the chemical and physical nature of the adsorbate $[39,40]$. Equation (2) can be rewritten to describe the total surface susceptibility of Pd deposits on the Au electrode surface as:

$$
\chi_{\mathrm{S}}^{(2)^{\prime}}=\chi_{\mathrm{Au}}^{(2)}+\chi_{\mathrm{Pd}}^{(2)}+\Delta \chi_{\mathrm{Pd}-\mathrm{Au}}^{(2)}
$$

where, $\chi_{\mathrm{Pd}}^{(2)}$ is the surface susceptibility of the $\mathrm{Pd}$ and $\Delta \chi_{\mathrm{Pd}-\mathrm{Au}}^{(2)}$ represents the perturbation in the nonlinear susceptibility due to the interaction between the deposited $\mathrm{Pd}$ and the $\mathrm{Au}$ substrate. The SHG intensity of the $\mathrm{Pd}$ electrode in $\mathrm{HClO}_{4}$ was found to be lower than that from the Au surface, i.e., $\chi_{\mathrm{Pd}}^{(2)}<\chi_{\mathrm{Au}}^{(2)}$, under visible excitation [63]. This relation was confirmed by the present study both for near-IR and visible excitations as the SHG intensities of the gold substrate with a very thick Pd layer were weaker than those of the bare $\mathrm{Au}$ under respective excitation. Thus, the contribution of $\chi_{\mathrm{Pd}}^{(2)}$ for the enhancement of the SHG signal can be ignored.

The measured SHG intensities are normalized with the potential dependent SHG intensities from the $\mathrm{Au}$ surface in contact with the $\mathrm{HClO}_{4}$ solution under the respective 
excitation wavelength. The potential dependent SHG response from the Au electrode in contact with $\mathrm{HClO}_{4}$ was first obtained, prior to $\mathrm{Pd}$ deposition, using the same experimental conditions as for Pd deposition. The normalized SHG signal was then square rooted to correlate with the nonlinear susceptibility as:

$$
\sqrt{I_{\mathrm{norm}}^{2 \omega}}=\sqrt{I_{\mathrm{Pd}-\mathrm{Au}}^{2 \omega} / I_{\mathrm{Au}}^{2 \omega}}
$$

By substituting Eq. 4 into Eq. 1, a simple relation between $\sqrt{I_{\text {norm }}^{2 \omega}}$ and $Q_{\mathrm{Pd}}$ is obtained as:

$$
\frac{\chi_{\mathrm{S}}^{(2)}}{\chi_{\mathrm{Au}}^{(2)}} \propto \sqrt{I_{\mathrm{norm}}^{2 \omega}}=\sqrt{\frac{I_{\mathrm{Pd}-\mathrm{Au}}^{2 \omega}}{I_{\mathrm{Au}}^{2 \omega}}}=1+c\left|Q_{\mathrm{Pd}}\right|
$$

where the proportionality constant, $c$, represents the $\Delta \chi_{\mathrm{Pd}-\mathrm{Au}}^{(2)}$ susceptibility and the deviations in the SHG signal from the Au substrate $\left(I_{\mathrm{Au}}^{2 \omega}\right)$.

Figure 4 shows the Pd coverage dependence of $\sqrt{I_{\text {norm }}^{2 \omega}}$ obtained under (a) near-IR and (b) visible excitations. Note $\sqrt{I_{\text {norm }}^{2 \omega}}=1$ means that the SHG intensity is equal to that from the bare $\mathrm{Au}$ electrode surface at a given potential without $\mathrm{Pd}$ or $\mathrm{PdCl}_{4}{ }^{2-}$.

The effect of the $\mathrm{PdCl}_{4}{ }^{2-}$ adsorption is clearly seen under near-IR excitation as shown in Fig. 4 (a). The $\sqrt{I_{\text {norm }}^{2 \omega}}$ value was larger than 1 with no Pd deposition, i.e., $Q_{\mathrm{Pd}}=0 \mu \mathrm{C}$ $\mathrm{cm}^{-2}$ due to the reason described in the previous section. $\sqrt{I_{\text {norm }}^{2 \omega}}$ decreased linearly with $Q_{\text {Pd }}$ during the initial stages of Pd deposition up to ca. $250 \mu \mathrm{C} \mathrm{cm}^{-2}$, and thereafter, a constant signal was observed irrespective of $Q_{\mathrm{Pd}}$. Under near-IR excitation, anion adsorption is often observed with an increase in the SHG signal [40].

Under visible excitation, initially $\sqrt{I_{\text {norm }}^{2 \omega}}$ linearly increased with $Q_{\mathrm{Pd}}$ but with two 
distinct slopes of $5.2 \times 10^{-3}$ and $1.7 \times 10^{-3} \mu \mathrm{C}^{-1} \mathrm{~cm}^{2}$ for $\mathrm{Q}_{\mathrm{Pd}}$ values from 0 to ca. $460 \mu \mathrm{C}$ $\mathrm{cm}^{-2}$ and from 460 to $920 \mu \mathrm{C} \mathrm{cm}^{-2}$, respectively. It then decreased for higher $Q_{\mathrm{Pd}}$ with the slope of $-5.4 \times 10^{-4} \mu \mathrm{C}^{-1} \mathrm{~cm}^{2}$. It is interesting to note that 460 and $920 \mu \mathrm{C} \mathrm{cm}^{-2}$ correspond

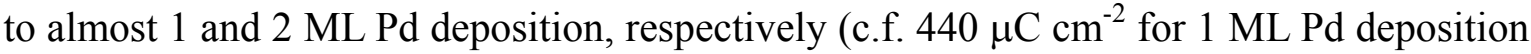
on an atomically flat $\mathrm{Au}(111)$ surface). Thus, the slope seemed to change when the $\mathrm{Pd}$ thickness was changed from 1,2, and $3 \mathrm{ML}$, suggesting the layer-by-layer growth of Pd that was found to occur on single-crystalline $\mathrm{Au}(111)[22,28]$ and $\mathrm{Au}(100)[25]$ electrode surfaces.

The results in Fig. 4 show that the SHG response under near-IR excitation was sensitive to only the initial sub-ML deposition of Pd but that under visible excitation was dependent on the Pd coverage over a wide region. This wavelength dependence can be explained by considering the schematic diagram of the density of states (DOS) shown in Fig. 5 for (a) a gold surface [69], (b) the Au surface with a Pd ML [54,55] and (c) a Pd surface [54].

(i) Near-IR excitation: As mentioned earlier, under near-IR excitation, a resonant SHG signal was observed at the Au surface as $2 \omega$ photons coupled with either the interband transition $(2.25 \mathrm{eV})$ from the upper edge of the $5 \mathrm{~d}$ band to the states near the Fermi level located in the $6 \mathrm{~s}$ band $[59,60]$ or an intraband transition within the sp band [65].

According to theoretical calculations for $1 \mathrm{ML} \mathrm{Pd}$ on a $\mathrm{Au}(111)$ slab [54,55], $\mathrm{Pd}$ 
deposition results in a shift of the d-band center towards the Fermi level as shown in Fig. 5(b). A UPS study [53] has also shown the upward shift of the d-band edge even with the sub-ML deposition of Pd on the Au surface.

When interband transition is considered to be the main source of resonant SHG signal from the Au surface, the shift in the d-band edge towards the Fermi level during the initial sub-ML deposition of Pd, an emergence of multiple transition between the $5 \mathrm{~d}$ and $6 \mathrm{~s}$ bands would be expected. Using simplified consideration, one may expect that the upward shift of the d-band edge give rise to a larger SHG signal, as more states are available for multiple transitions near the Fermi level. However, quite opposite results were experimentally observed for the SHG measurements from the Pd and Pt transition metal electrode surfaces in contact with $\mathrm{HClO}_{4}$ solution in the same potential region of interest [63]. The SHG intensity obtained from the Pd and Pt surfaces, whose Fermi level situate within the d-band, was weaker than that from the Au surface whose d-band is far below the Fermi level. Since the d-band widths of transition metals (Pd: ca. 6 eV [54], Pt: ca. 8 eV [69]) are greater than the coupling resonance energy, multiple transitions from the numerous states in the d-band to the empty states above the Fermi level are possible. These multiple transitions are prone to interference with one another, resulting in a weaker SHG signal than that from the Au surface where the SHG signal observed occurs as a result of specific resonance. 
On the other hand, if the resonant SHG signal from $\mathrm{Au}$ was a result of intraband transition within the sp band, the shift in the d-band toward the Fermi level can cause scattering of free electrons within the sp band. This leads to a decrease in the SHG signal for surfaces when the d-band nears the Fermi level.

The origin for the resonant SHG signal from Au is still speculative, whether it arises as a result of coupling with either the interband or intraband transition. However, in either case, the change in the surface electronic structure with the d-band edge shift can explain the decrease in the SHG signal during the initial sub-ML deposition of Pd and the constant SHG response after the up shift in the d-band with increased Pd surface coverage.

(ii) Visible excitation: In contrast to the above results, an enhancement in $\sqrt{I_{\text {norm }}^{2 \omega}}$ was initially observed with $\mathrm{Pd}$ deposition on the $\mathrm{Au}$ substrate under visible excitation. This enhancement in the SHG signal can be explained by a direct coupling of a photon with an electronic transition at the Pd deposited gold with the modified electronic structure. The resonant enhancement may arise from the coupling of either the $\omega_{\mathrm{VIS}}(2.06 \mathrm{eV})$ or $2 \omega_{\mathrm{VIS}}$ $(4.13 \mathrm{eV})$ photon with an electronic transition either from an occupied bound state to the states around the Fermi level or from the Fermi level to an unoccupied energy state.

A UPS study of the Au (111) surface with 1ML Pd has shown the formation of an induced hybrid state below the Fermi level positioned at $-3.5 \mathrm{eV}$ as a result of the $\mathrm{Pd}$ 4d-Au 5d interaction [53]. A similar hybrid state positioned at $-4.2 \mathrm{eV}$ has been also 
reported for the Pd-Au alloy system [70]. Thus, there is a possibility of direct coupling of the $2 \omega_{\mathrm{VIS}}$ photon with the electronic transition from the hybrid state to the Fermi level. However, it is very unlikely because the localized electron density of the hybrid state is comparable to other band features located near this state and is rather small [53]. The possibility of resonant enhancement due to the coupling of the $\omega$ and $2 \omega$ photons with the electronic transition of the bound electrons can be excluded.

Another possible origin for the SHG enhancement is the resonance of either the $\omega_{\mathrm{VIS}}$ or $2 \omega_{\mathrm{VIS}}$ photons with an electronic transition from the Fermi level to an unoccupied energy state located at ca. 2 or $4 \mathrm{eV}$, respectively, above the Fermi level. Such states are shown by the dashed lines in Fig. 5(b). The image state is one possibility as the final state for the coupled transition. The image state $(n=1)$ of $\mathrm{Au}(111)$ was confirmed by Two Photon Photoemission spectroscopic study (TPPS) to be located at ca. $4.7 \mathrm{eV}$ [71] above the Fermi level. Although, the image state is positioned at too high energy to be responsible for the present resonant features $(2 \omega=4.13 \mathrm{eV})$, the energy of the image states became lower at the Pd deposited Au as was reported for the Au deposited Pd(111) surface, where the image state of Pd(111), positioned at ca. $4.9 \mathrm{eV}$ [72] shifted near the Fermi level with increasing Pd thickness [73]. However, since the theoretical calculation of the band structure above the Fermi level for the Au surface with a sub-ML of Pd has not yet been carried out, the exact energy level of these states are not known. SHG spectroscopy at single-crystalline Au 
electrodes is currently underway to obtain quantitative information on the electronic transitions involved.

\subsubsection{Bulk Pd deposition}

The SHG signal under visible excitation decreased with $Q_{\mathrm{Pd}}$ or the Pd thickness when the Pd thickness was more than $3 \mathrm{ML}$ as shown in Fig. 4(b). The relation between $\sqrt{I_{\text {norm }}^{2 \omega}}$ and $Q_{\mathrm{Pd}}$ or the Pd thickness for a wider range of $Q_{\mathrm{Pd}}$ derived from Fig. 3 is shown in Fig. 6.

It is clear that $\sqrt{I_{\text {norm }}^{2 \omega}}$ exponentially decreased with the Pd thickness. This behavior can be simply explained by considering that the SHG takes place only at the Pd/Au interface and both the excitation and $\mathrm{SH}$ photons are attenuated due to the absorption by the Pd layer. The light intensity at the $\mathrm{Pd} / \mathrm{Au}$ interface, $I_{\mathrm{z}}$, with an angle of incidence, $\theta$, is given by [74]:

$$
I_{\mathrm{z}}=I_{0}^{\omega} \exp \left(\frac{-\alpha^{\omega} d}{\cos \theta}\right)
$$

where $\alpha^{\omega}$ is the absorption coefficient of the excitation light in $\mathrm{Pd}, d$ is the thickness of the Pd overlayer, and $I_{0}^{\omega}$ is the intensity of the incident light at the surface of the Pd overlayer. The intensity of the SH light generated at the $\mathrm{Pd} / \mathrm{Au}$ interface is proportional to the square of $I_{\mathrm{Z}}$ as described by Eq. 1. The SHG intensity at the surface of Pd overlayer, $I_{\mathrm{Pd}-\mathrm{Au}}^{2 \omega}$, can be given by: 


$$
I_{\mathrm{Pd}-\mathrm{Au}}^{2 \omega} \propto\left(I_{0}^{\omega}\right)^{2} \exp \left(\frac{-\alpha^{\omega} d}{\cos \theta}\right)^{2} \cdot \exp \left(\frac{-\alpha^{2 \omega} d}{\cos \theta}\right)
$$

where $\alpha^{2 \omega}$ is the absorption coefficient of SH light in Pd. Here, it is assumed that the light path of the SH light is equal to that of the excitation light, $d / \cos \theta$, although they are slightly different. $I_{\mathrm{Pd}-\mathrm{Au}}^{2 \omega}$ was normalized with the SHG intensity from the Au surface $I_{\mathrm{Au}}^{2 \omega}$ as described by Eq. 5 and square rooted to obtain $\sqrt{I_{\text {norm }}^{2 \omega}}$ as a function of the Pd overlayer thickness as:

$$
\sqrt{I_{\text {norm }}^{2 \omega}} \propto I_{0}^{\omega} \exp \left(\frac{-d\left(2 \alpha^{\omega}+\alpha^{2 \omega}\right)}{2 \cos \theta}\right)
$$

The calculated result using the values of $\theta=45^{\circ}, \alpha^{\omega}=0.043 \mathrm{~nm}^{-1}$ and $\alpha^{2 \omega}=0.048 \mathrm{~nm}^{-1}$ [75] is plotted in Fig. 6 by a solid curve. Fairly good agreement was obtained between the experimental results and the calculated curve as long as the Pd thickness is more than ca. 2 $\mathrm{nm}$. It is reasonable that this simple approach cannot explain the thickness dependence of the SHG intensity when the Pd layer is very thin because the thickness dependent electronic structure change, i.e., the contribution of $\Delta \chi_{\mathrm{Pd}-\mathrm{Au}}^{(2)}$, is still important and the optical property of the Pd overlayer should be different from that of the bulk Pd in this region.

\section{Conclusions}

The Pd electrodeposition process on a polycrystalline $\mathrm{Au}$ electrode surface was 
investigated by SHG measurements under both near-IR $(1064 \mathrm{~nm})$ and visible $(600 \mathrm{~nm})$ excitations. Under near-IR excitation, the resulting SHG signal contained contributions from the $\mathrm{Au}$ substrate, the $\mathrm{Pd}$ deposit and the adsorbed $\mathrm{PdCl}_{4}{ }^{2-}$. On the other hand, the $\mathrm{PdCl}_{4}{ }^{2-}$ adsorption was found to have a negligible effect on the SHG signal under visible excitation. The SHG signal under visible excitation was resonantly enhanced with the first and second monolayer depositions of Pd but declined with higher Pd coverage. The origin of the resonant enhancement of the SHG signal is considered to arise from the coupling of the fundamental or SH photon with the interband transition from the Fermi level to a newly formed unoccupied state of higher energy. The SHG signal was found to exponentially decay with Pd thickness during the Pd bulk deposition as a result of the absorption of the exciting and SH photons by Pd layers.

\section{Acknowledgements}

This work was partially supported by Grants-in-Aid for Scientific Research from the Ministry of Education, Science, Sports and Culture, Japan. TA acknowledges the Japan Society for the Promotion of Science for the Fellowship for Young Scientists. We would also like to thank Drs. H. Naohara and T. Kondo for their valuable comments and discussions. 


\section{References}

[1] G.A. Somorjai, Introduction to Surface Chemistry and Catalysis, Wiley, New York, 1994.

[2] C.R. Henry, Surf. Sci. Rep. 31 (1998) 235.

[3] J.A. Rodriguez, Surf. Sci. Rep. 24 (1996) 223.

[4] J.H. Sinfelt, Bimetallic Catalysts: Discoveries, Concepts and Applications, Wiley, New York, 1983.

[5] E.G. Allison, G.C. Bond, Catal. Rev. 7 (1972) 233.

[6] B.E. Koel, A. Sellidj, M.T. Paffett, Phys. Rev. B 46 (1992) 7846.

[7] X. Shen, D.J. Frankel, J.C. Hermanson, G.J. Lapeyre, R.J. Smith, Phys. Rev. B 32 (1985) 2120.

[8] S. Xinyin, D.J. Frankel, G.J. Lapeyre, R.J. Smith, Phys. Rev. B 33 (1986) 5372.

[9] D. Cherns, M.J. Stowell, Thin Solid Films 29 (1975) 127.

[10] D. Cherns, M.J. Stowell, Thin Solid Films 29 (1975) 107.

[11] C.J. Baddeley, C.J. Barnes, A. Wander, R.M. Ormerod, R.M. Lambert, Surf. Sci. 314 (1994) 1.

[12] C.J. Baddeley, M. Tikhov, C. Hardacre, J.R. Lomas, R.M. Lambert, J. Phys. Chem. 100 (1996) 2189.

[13] G.A. Attard, A. Bannister, J. Electroanal. Chem. 300 (1991) 467.

[14] N.M. Markovic, C.A. Lewis, V. Climent, V. Stamenkovic, P.N. Ross, Surf. Sci. $465(2000) 103$.

[15] B. Álvarez, A. Rodes, J.M. Pérez, J.M. Feliu, J.L. Rodríguez, E. Pastor, Langmuir $16(2000) 4695$.

[16] K. Uosaki, S. Ye, H. Naohara, Y. Oda, T. Haba, T. Kondo, J. Phys. Chem. B 101 
(1997) 7566.

[17] S. Gonda, Handbook of the Thin Layer for Preparation, Evaluation and Application, Fuji Technosystems, Tokyo, 1986.

[18] A.A. Gewirth, B.K. Niece, Chem. Rev. 97 (1997) 1129.

[19] J.R. LaGraff, A.A. Gewirth, in: H. Siegenthaler (Ed.), Nanoscale Probes of Solid/Liquid Interfaces, Kulwer, Dordrecht, 1995, pp 83.

[20] K. Uosaki, M. Koinuma, N. Sekine, S. Ye, in: G. Jerkoiewicz, M.P. Soriaga, K. Uosaki, A. Wieckowski (Eds.), Solid-Liquid Electrochemical Interfaces, American Chemical Society, Washington, D.C., 1997, pp 189.

[21] L.A. Kibler, R. Kleinert, R. Randler, D.M. Kolb, Surf. Sci. 443 (1999) 19.

[22] H. Naohara, S. Ye, K. Uosaki, J. Phys. Chem. B 102 (1998) 4366.

[23] H. Naohara, S. Ye, K. Uosaki, Coll. and Surf. A 154 (1999) 201.

[24] H. Naohara, S. Ye, K. Uosaki, Appl. Phys. A 66 (1998) S457.

[25] H. Naohara, S. Ye, K. Uosaki, J. Electroanal. Chem. 473 (1999) 2.

[26] M. Baldauf, D.M. Kolb, Electrochim. Acta 38 (1993) 2145.

[27] L.A. Kibler, M. Kleinert, D.M. Kolb, Surf. Sci. 461 (2000) 155.

[28] M. Takahasi, Y. Hayashi, J. Mizuki, K. Tamura, T. Kondo, H. Naohara, K. Uosaki, Surf. Sci. 461 (2000) 213.

[29] M. Takahasi, Y. Hayashi, J. Mizuki, K. Tamura, T. Kondo, H. Naohara, K. Uosaki, submitted.

[30] H. Naohara, S. Ye, K. Uosaki, unpublished data.

[31] M. Baldauf, D.M. Kolb, J. Phys. Chem. 100 (1996) 11375.

[32] H. Naohara, S. Ye, K. Uosaki, Electrochim. Acta 45 (2000) 3305.

[33] K. Shimazu, H. Kita, J. Catal. 83 (1983) 393. 
[34] K. Shimazu, H. Kita, J. Catal. 83 (1983) 414.

[35] K. Gossner, E. Mizera, J. Electroanal. Chem. 125 (1981) 359.

[36] B. Álvarez, V. Climent, J.M. Feliu, A. Aldaz, Electrochem. Comm. 2 (2000) 427.

[37] H. Naohara, S. Ye, K. Uosaki, J. Electroanal. Chem. 500 (2001) 435.

[38] P. Guyot-Sionnest, W. Chen, Y.R. Shen, Phys. Rev. B 33 (1986), 8254.

[39] Y.R. Shen, The Principle of Nonlinear Optics, Wileys, New York, 1984.

[40] R.M. Corn, D.A. Higgins, Chem. Rev. 94 (1994) 107.

[41] T.F. Heinz, in: H.E. Ponath, G.I. Stegeman (Eds.), Nonlinear Surface Electromagnetic Phenomena, Elsevier, Amsterdam, 1991, Ch. 5.

[42] G.L. Richmond, J.M. Robinson, V.L. Shannon, Prog. Surf. Sci. 28 (1988) 1.

[43] R.A. Georgiadis, G.A. Neff, G.L. Richmond, J. Chem. Phys. 92 (1990) 4623.

[44] J.F. McGlip, Prog. Surf. Sci. 49 (1995) 1.

[45] G. Lüpke, Surf. Sci. Rep. 35 (1999) 75.

[46] G. Lüpke, D.J. Bottomley, H.M. van Driel, Phys. Rev. B 49 (1994) 2104.

[47] G. Petrocelli, S. Martellucci, R. Francini, Appl. Phys. Rev. A 56 (1993) 263.

[48] K.B. Eisenthal, Annu. Rev. Phys. Chem. 43 (1992) 627.

[49] R.R. Naujok, D.A. Higgins, D.G. Hanken, R.M. Corn, J. Chem. Soc., Faraday Trans. 91 (1995) 1411.

[50] T. Rasing, Y.R. Shen, Phys. Rev. Lett. 55 (1985) 2903.

[51] I. Yagi, S. Nakabayashi, K. Uosaki, J. Phys. Chem. B 101 (1997) 7414.

[52] I. Yagi, K. Uosaki, in preparation.

[53] A. Sellidj, B.E. Koel, Phy. Rev. B 49 (1994) 8367.

[54] V. Pallassana, M. Neurock, J. Catal. 191 (2000) 301.

[55] A. Ruban, B. Hammer, P. Stoltz, H.L. Skriver, J.K. Nørskov, J. Mol. Catal. A: 
Chem. 115 (1997) 421.

[56] I. Yagi, J.M. Lantz, S. Nakabayashi, R.M. Corn, K. Uosaki, J. Electroanal. Chem. $401(1996) 95$.

[57] I. Yagi, S. Nakabayashi, K. Uosaki, Surf. Sci. 406 (1998) 1.

[58] I. Yagi, S. Nakabayashi, K. Uosaki, J. Phys. Chem. B 102 (1998) 2677.

[59] P. Guyot-Sionnest, A. Tadjeddine, J. Chem. Phys. 92 (1990) 734.

[60] E.W.L. Wong, G.L. Richmond, J. Chem. Phys. 99 (1993) 5500.

[61] N. Bloembergen, R.K. Chang, S.S. Jha, C.H. Lee, Phys. Rev. 174 (1968) 813.

[62] G.L. Richmond, Langmuir 2 (1986) 132.

[63] T. Awatani, I. Yagi, K. Uosaki, unpublished data

[64] D.M. Kolb, J.D.E. McIntyre, Surf. Sci. 28 (1971) 321.

[65] W. Hübner, K.H. Bennemann, K. Böhmer, Phys. Rev. B, 50 (1994) 17597.

[66] M. Buck, F. Eisert, M. Grunze, F. Träger, Ber. Bunsenges. Phys. Chem. 97 (1993) 399.

[67] M. Buck, F. Eisert, M. Grunze, J. Fisher, F. Träger J. Vac. Sci. Technol. A 10 (1992) 926.

[68] H. Rieley, N.J. Price, R.G. White, R.I.R. Blyth, A.W. Robinson, Surf. Sci. 331 (1995) 189.

[69] B. Hammer, J.K. Norskov, Nature (1995) 376.

[70] D.L. Weissman-Wenocur, P.M. Stefan, B.B. Pate, M.L. Shek, I. Lindau, W.E. Spicer, Phys. Rev. B 27 (1983) 3308.

[71] R. Fischer, S. Schuppler, N. Fischer, Th. Fauster, W. Steinmann, Phys. Rev. Lett. 70 (1993) 654.

[72] D. Straub, F.J. Himpsel, Phys. Rev. B, 33 (1986) 2256. 
[73] R. Fischer, T. Faustner, Phys. Rev. B, 51 (1995) 7112.

[74] M. Born, E. Wolf, Principles of Optics, Fifth Ed., Pergamon Press, 1975.

[75] E.D. Palik, Handbook of Optical Constants of Solids II, Academic Press, 1985. 


\section{Figures}

Fig. 1. Solid line shows the potential dependence of (a) the current and (b) the near-IR (SHG: $532 \mathrm{~nm}$ ) and (c) the visibly excited (SHG: $300 \mathrm{~nm}$ ) SHG signal from a polycrystalline $\mathrm{Au}$ electrode in a $0.1 \mathrm{M} \mathrm{HClO}_{4}$ aqueous solution containing $0.1 \mathrm{mM}$ $\mathrm{PdCl}_{4}{ }^{2-}$. The dotted line shows the response obtained from an aqueous solution containing only $0.1 \mathrm{M} \mathrm{HClO}_{4}$. Sweep rate: $10 \mathrm{mV} / \mathrm{s}$; polarization: $\mathrm{p}$-in/p-out; input energy: $1 \mathrm{~mJ} /$ pulse; rep. rate: $20 \mathrm{~Hz}$.

Fig. 2. Time dependent plots over two potential cycles (solid line - first cycle; dotted line - second cycle) of (a) the potential, (b) the SHG signal and (c) the current of a polycrystalline $\mathrm{Au}$ electrode in a $0.1 \mathrm{M} \mathrm{HClO}_{4}$ aqueous solution containing $0.1 \mathrm{mM}$ $\mathrm{PdCl}_{4}{ }^{2-}$ under near-IR (SHG: $532 \mathrm{~nm}$ ) excitation. Sweep rate: $10 \mathrm{mV} / \mathrm{s}$; polarization: p-in/p-out; input energy: $1 \mathrm{~mJ} /$ pulse; rep. rate: $20 \mathrm{~Hz}$.

Fig. 3. Time dependent plots of (a) the potential, (b) the current and (c) the SHG signal during a large electrodeposition of $\mathrm{Pd}$ on the $\mathrm{Au}$ surface in a $0.1 \mathrm{M} \mathrm{HClO}_{4}$ aqueous solution containing $0.5 \mathrm{mM} \mathrm{PdCl}_{4}{ }^{2-}$ under visible (SHG: $300 \mathrm{~nm}$ ) excitation. Sweep rate: $5 \mathrm{mV} / \mathrm{s}$; polarization: p-in/p-out; input energy: $1 \mathrm{~mJ} /$ pulse; rep. rate: $20 \mathrm{~Hz}$.

Fig. 4. Relation between square root of the normalized SHG $\left(\sqrt{I_{\text {norm }}^{2 \omega}}\right)$ of (a) $532 \mathrm{~nm}$ and (b) $300 \mathrm{~nm}$ intensities and the Faradaic charge associated with Pd electrodeposition 
$\left(Q_{\mathrm{Pd}}\right)$ onto the $\mathrm{Au}$ electrode in a $0.1 \mathrm{M} \mathrm{HClO}_{4}$ aqueous solution containing $0.5 \mathrm{mM}$ $\mathrm{PdCl}_{4}{ }^{2-}$. Solid lines are linear least-square fits.

Fig. 5. Schematic diagram of (a) Au, (b) 1 ML deposited Pd on Au and (c) Pd surface density of states (DOS).

Fig. 6. Relation between square root of the normalized SHG (300 nm) intensity $\left(\sqrt{I_{\text {norm }}^{2 \omega}}\right)$ and the Faradaic charge associated with Pd electrodeposition $\left(Q_{\mathrm{Pd}}\right)$ onto the $\mathrm{Au}$ electrode in a $0.1 \mathrm{M} \mathrm{HClO}_{4}$ aqueous solution containing $0.5 \mathrm{mM} \mathrm{PdCl}_{4}{ }^{2-}$. The top x-axis shows the equivalent Pd thickness $(d)$ evaluated from $Q_{\text {Pd }}$. The data points (open circles) have been averaged over 50 points for clarity. The solid line is a fit using Eq. 9 . 
Avatani et al., Fi gure 1

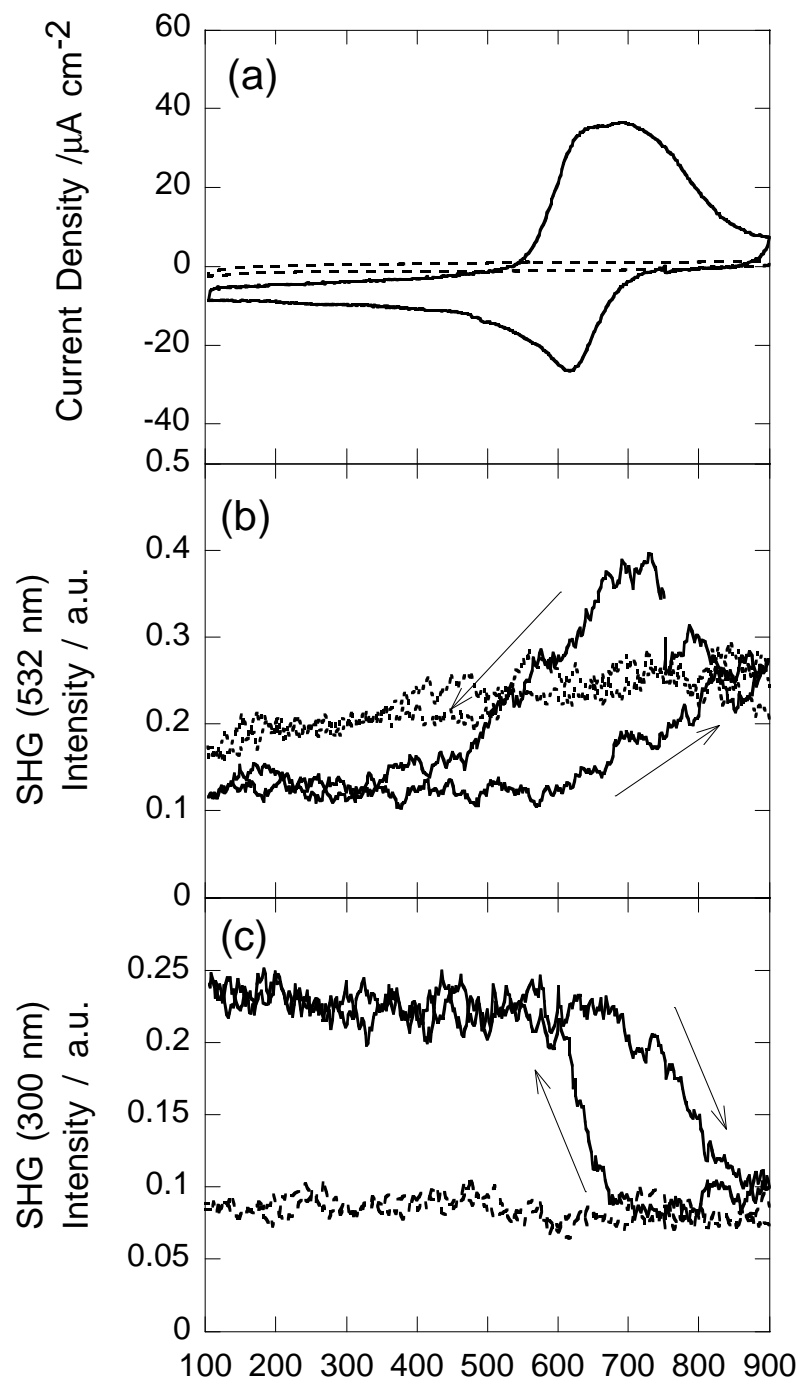

Potential / $\mathrm{mV}$ vs $\mathrm{Ag}|\mathrm{AgCl}| \mathrm{NaCl}$ (sat) 
Avatani et al., Fi gure 2

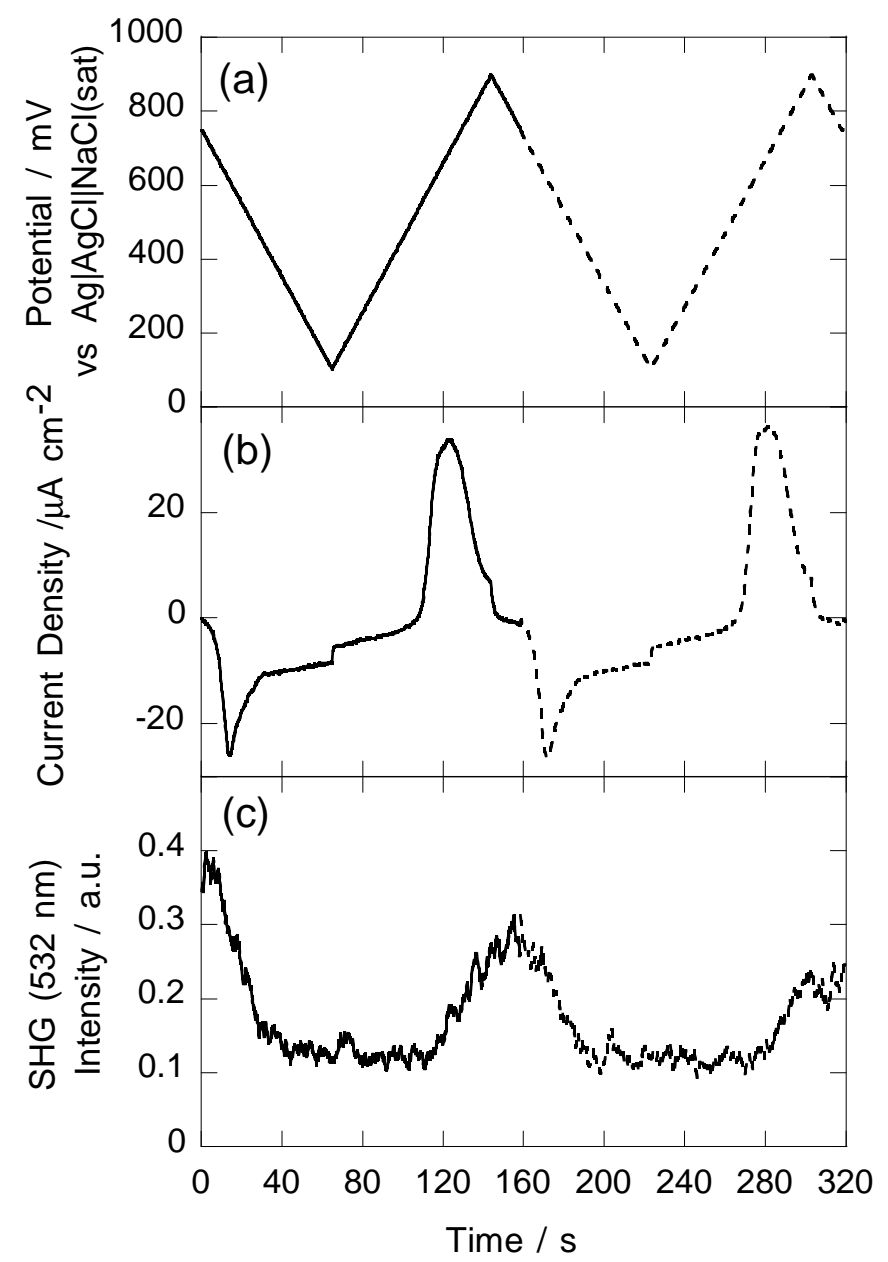


Awatani et al., Fi gure 3

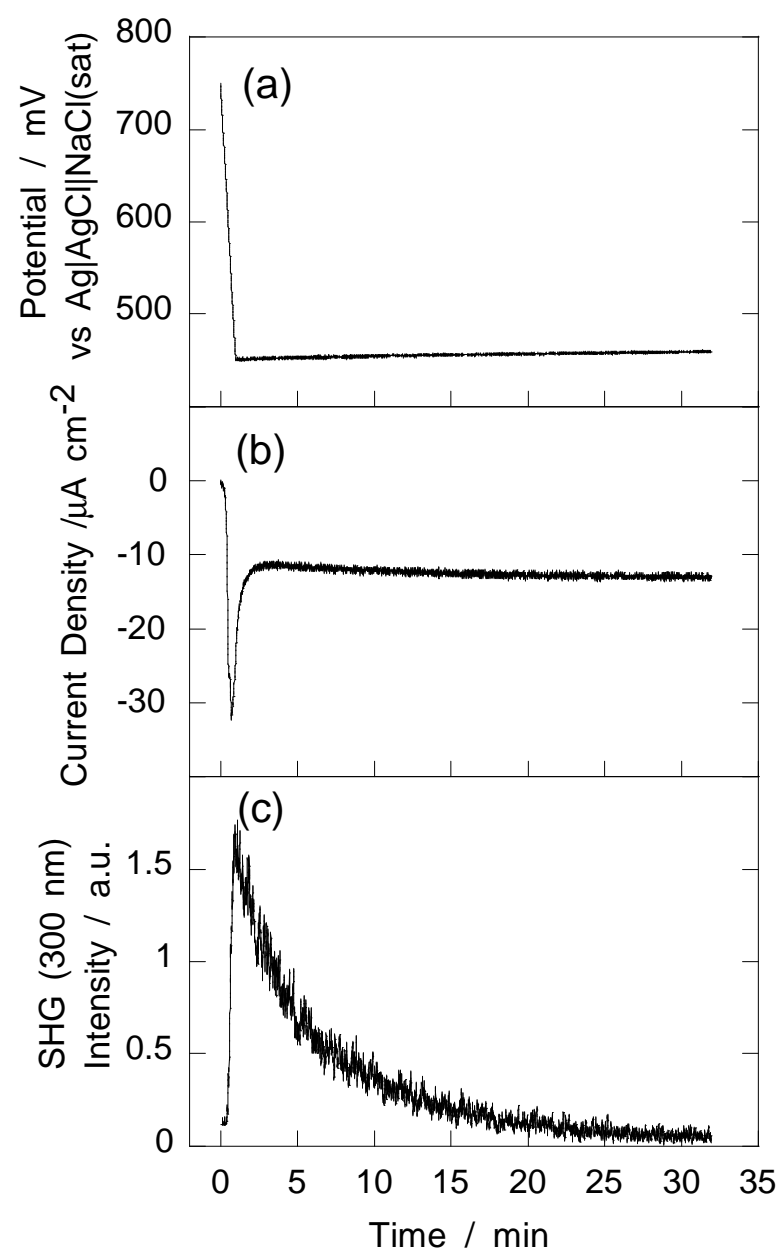


Avatani et al., Fi gure 4

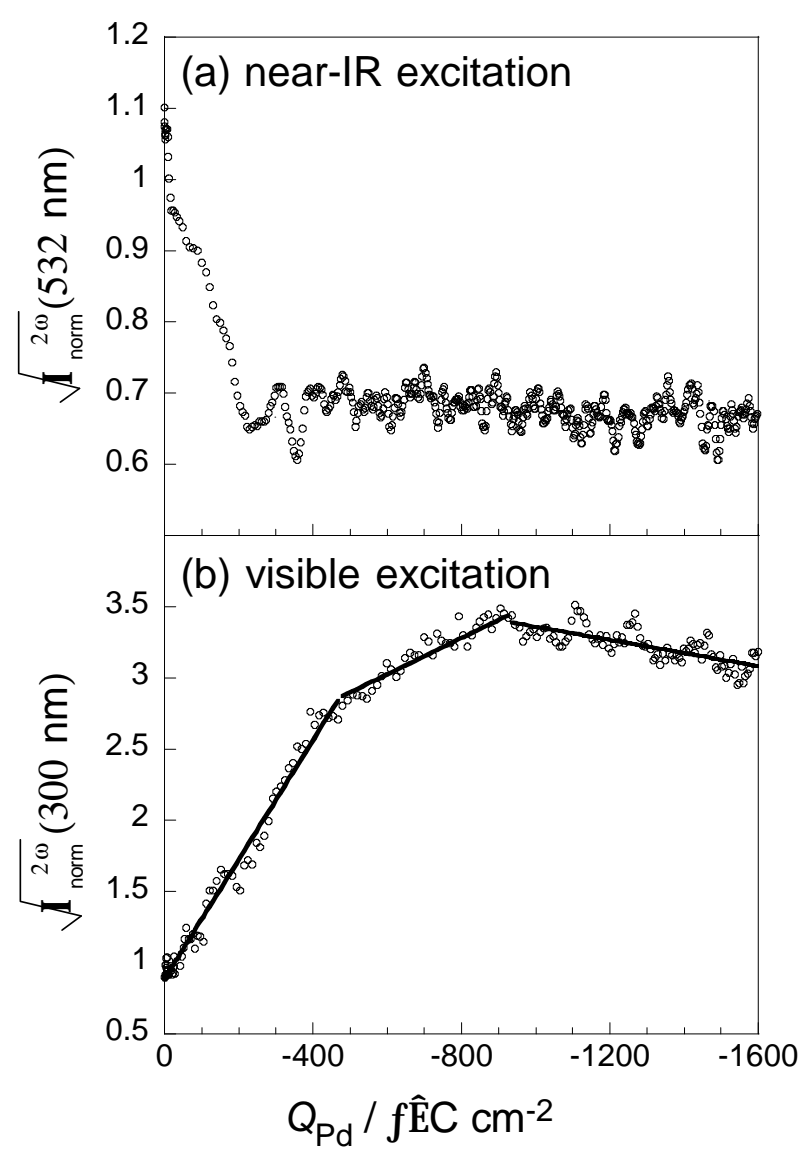


Awatani et al., Fi gure 5

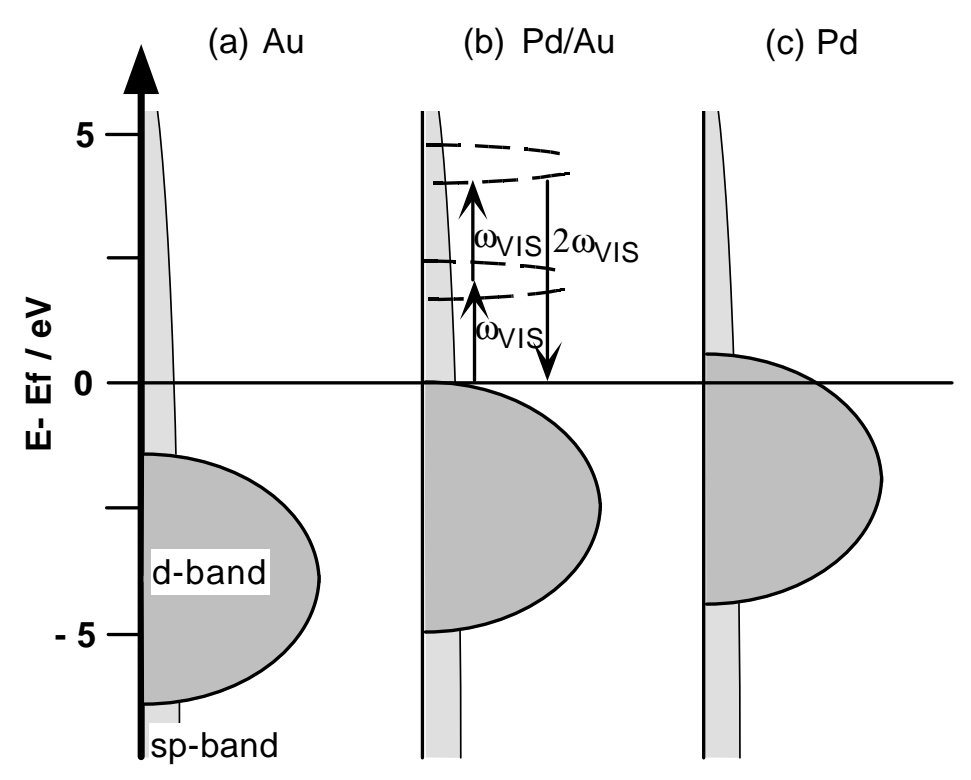


Amat ani et al., Fi gure 6

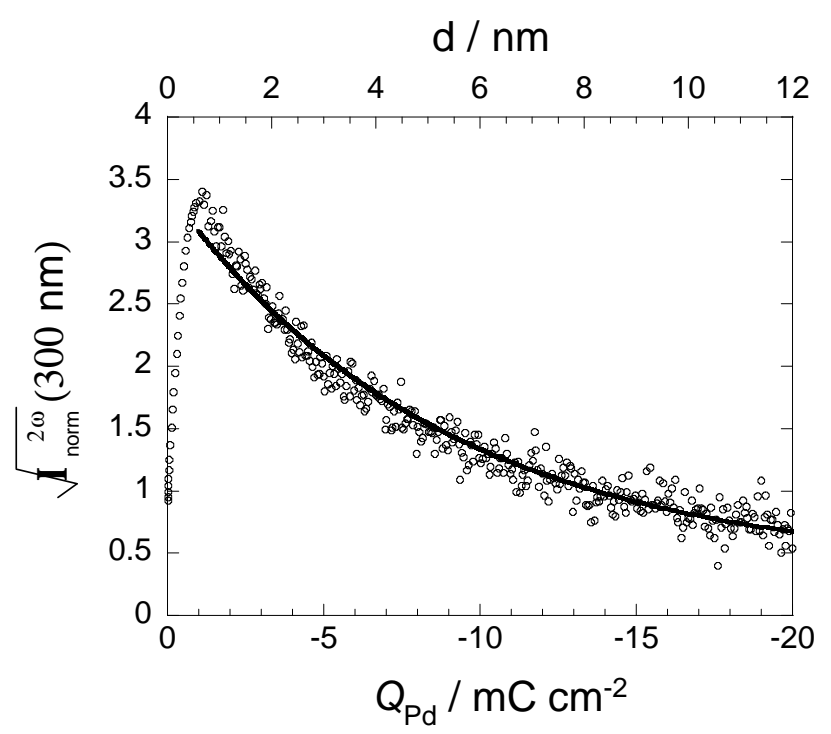

